\title{
ON SIMPLE MODULES OVER TWISTED FINITE CATEGORY ALGEBRAS
}

\author{
MARKUS LINCKELMANN AND MICHA£ STOLORZ
}

(Communicated by Pham Huu Tiep)

\begin{abstract}
The purpose of this paper is to show that the recent proof by Ganyushkin, Mazorchuk and Steinberg of the parametrisation of simple modules over finite semigroup algebras due to Clifford, Munn and Ponizovskiĭ carries over to twisted finite category algebras. We observe that the parametrisations of simple modules over Brauer algebras, Temperley-Lieb algebras, and Jones algebras due to Graham and Lehrer can be obtained as special cases of our main result. We further note that the notion of weights in the context of Alperin's weight conjecture extends to twisted finite category algebras.
\end{abstract}

\section{INTRODUCTION}

Let $k$ be a commutative ring and $\mathcal{C}$ a small category. The set of idempotents in the morphism set $\operatorname{Mor}(\mathcal{C})$ of $\mathcal{C}$ is partially ordered via $e \leq f$ if $e, f$ are idempotent endomorphisms of the same object in $\mathcal{C}$ such that $e=e \circ f=f \circ e$. For any idempotent endomorphism $e$ of an object $X$ in $\mathcal{C}$ we denote by $G_{e}$ the group of invertible elements in the monoid $e \circ \operatorname{End}_{\mathcal{C}}(X) \circ e$. Two idempotent endomorphisms $e \in \operatorname{End}_{\mathcal{C}}(X)$ and $f \in \operatorname{End}_{\mathcal{C}}(Y)$ are called isomorphic if there are morphisms $s \in$ $f \circ \operatorname{Hom}_{\mathcal{C}}(X, Y) \circ e$ and $t \in e \circ \operatorname{Hom}_{\mathcal{C}}(Y, X) \circ f$ such that $t \circ s=e$ and $s \circ t=f$. The elements $s$ and $t$ determine a group isomorphism $G_{e} \cong G_{f}$, sending $u \in G_{e}$ to $s \circ u \circ t$, with inverse sending $v \in G_{f}$ to $t \circ v \circ s$. A different choice of $s, t$ will lead to a possibly different group isomorphism, but any two different choices will yield group isomorphisms which differ at most by an inner automorphism of $G_{e}$. If $U$ is a $k \mathcal{C}$-module, then $e U$ is a module for the monoid algebra $k\left(e \circ \operatorname{End}_{\mathcal{C}}(X) \circ e\right)$, hence restricts to a module over the group algebra $k G_{e}$. Two pairs $(e, U),(f, V)$ consisting of idempotent endomorphisms $e \in \operatorname{End}_{\mathcal{C}}(X), f \in \operatorname{End}_{\mathcal{C}}(Y)$, a $k G_{e}$-module $U$ and a $k G_{f}$-module $V$ are called isomorphic if there are morphisms $s \in f \circ \operatorname{Hom}_{\mathcal{C}}(X, Y) \circ e$ and $t \in e \circ \operatorname{Hom}_{\mathcal{C}}(Y, X) \circ f$ such that $t \circ s=e$ and $s \circ t=f$, and such that the isomorphism classes of $U, V$ correspond to each other through the algebra isomorphism $k G_{e} \cong k G_{f}$ induced by $s$ and $t$. Since inner automorphisms of an algebra stabilise all isomorphism classes of modules, this property is independent of the choice of $s$ and $t$.

Theorem 1.1. Let $k$ be a commutative ring and $\mathcal{C}$ a finite category. The map sending a simple $k \mathcal{C}$-module $S$ to the pair $(e, e S)$, where $e$ is an idempotent endomorphism in $\mathcal{C}$, minimal with respect to $e S \neq\{0\}$, induces a bijection between the set of isomorphism classes of simple $k \mathcal{C}$-modules and the set of isomorphism classes

Received by the editors April 26, 2011.

2010 Mathematics Subject Classification. Primary 16D60, 18B40. 
of pairs $(e, T)$ consisting of an idempotent endomorphism e in $\mathcal{C}$ and a simple $k G_{e^{-}}$ module $T$.

For semigroup algebras this is due to Clifford [3], 4, Munn [16] and Ponizovskiu [17. If $\mathcal{C}$ is a finite $E I$-category (that is, if all endomorphisms of objects are isomorphisms), the above parametrisation specialises to the parametrisation, due to Lück, in terms of simple modules of automorphism groups of objects (see [5. $\S 11, \mathrm{~B}]),[15, \S 9]$, or also [23, 4.3]). For inverse semigroup algebras and inverse category algebras this follows also from 21] and [14. Our proof of Theorem 1.1 follows closely the lines of that given in [6] for semigroups, which has motivated the present work. As in [6], this is based on Green's work on Schur functors in [10, 6.2]. More precisely, if $e$ is an idempotent in a $k$-algebra $A$, then for any simple $A$-module $S$, either $e S$ is zero or $e S$ is a simple $e A e$-module. This induces a bijection between the set of isomorphism classes of simple $A$-modules not annihilated by $e$ and the set of isomorphism classes of simple $e A e$-modules. If $T$ is a simple $e A e$-module, then $A e \otimes_{e A e} T$ has a unique simple quotient and $\operatorname{Hom}_{e A e}(e A, T)$ has a unique simple submodule, both isomorphic to a simple $A$-module $S$ satisfying $e S \cong T$. This yields two explicit descriptions of the inverse map from the set of isomorphism classes of pairs $(e, T)$ as in Theorem 1.1 to the set of isomorphism classes of simple $k \mathcal{C}$-modules.

The methods work more generally for twisted finite category algebras. In order to describe this, let $\mathcal{C}$ be a finite category, $k$ a commutative ring and $\alpha$ a 2-cocycle of $\mathcal{C}$ with coefficients in $k^{\times}$. That is, if $s, t$ are morphisms in $\mathcal{C}$ for which the composition $t \circ s$ is defined, then $\alpha(t, s) \in k^{\times}$such that if $s, t, u$ are three morphisms in $\mathcal{C}$ for which the compositions $t \circ s$ and $u \circ t$ are defined, we have the 2 -cocycle identity $\alpha(u \circ t, s) \alpha(u, t)=\alpha(u, t \circ s) \alpha(t, s)$. As a $k$-module, the twisted category algebra $k_{\alpha} \mathcal{C}$ is equal to $k \mathcal{C}$, endowed with the $k$-bilinear multiplication $t \cdot s=\alpha(t, s) t \circ s$ if $t \circ s$ is defined, and $t \cdot s=0$ if not. The associativity of the multiplication in $k_{\alpha} \mathcal{C}$ is equivalent to the 2-cocycle identity. The isomorphism class of $k_{\alpha} \mathcal{C}$ depends only on the class of $\alpha$ in $H^{2}\left(\mathcal{C} ; k^{\times}\right)$. If $e$ is an idempotent endomorphism of an object $X$ in $\mathcal{C}$, the image of $e$ in $k_{\alpha} \mathcal{C}$ need no longer be an idempotent, because $e \cdot e=\alpha(e, e)(e \circ e)=\alpha(e, e) e$. Dividing this equation by $\alpha(e, e)^{2}$ shows that $\hat{e}=\alpha(e, e)^{-1} e$ is an idempotent in $k_{\alpha} \mathcal{C}$. We use the same letter $\alpha$ for the restriction of $\alpha$ to the monoid $e \circ \operatorname{End}_{\mathcal{C}}(X) \circ e$ and its subgroup $G_{e}$. We have $\hat{e} \cdot k_{\alpha} \mathcal{C} \cdot \hat{e}=k_{\alpha}\left(e \circ \operatorname{End}_{\mathcal{C}}(X) \circ e\right)$; in particular, the twisted group algebra $k_{\alpha} G_{e}$ is a unitary subalgebra of $\hat{e} \cdot k_{\alpha} \mathcal{C} \cdot \hat{e}$. Therefore, if $U$ is a $k_{\alpha} \mathcal{C}$-module, then $\hat{e} U$ is a $k_{\alpha} G_{e}$-module. We will see in Propositions 5.2 and 5.4 below that if $e, f$ are isomorphic idempotents in $\mathcal{C}$, there is an algebra isomorphism $k_{\alpha} G_{e} \cong k_{\alpha} G_{f}$ which is uniquely determined up to an inner automorphism. Hence, as before, two pairs $(e, U),(f, V)$ consisting of idempotents $e \in \operatorname{End}_{\mathcal{C}}(X), f \in \operatorname{End}_{\mathcal{C}}(Y)$, a $k_{\alpha} G_{e^{-}}$ module $U$ and a $k_{\alpha} G_{f}$-module $V$, are called isomorphic if $e, f$ are isomorphic and if the isomorphism classes of $U, V$ correspond to each other through the induced isomorphism $k_{\alpha} G_{e} \cong k_{\alpha} G_{f}$. With this notation, Theorem 1.1 can be generalised as follows.

Theorem 1.2. Let $k$ be a commutative ring and $\mathcal{C}$ a finite category. Let $\alpha$ be a 2cocycle of $\mathcal{C}$ with coefficients in $k^{\times}$. The map sending a simple $k_{\alpha} \mathcal{C}$-module $S$ to the pair $(e, \hat{e} S)$, where $e$ is an idempotent endomorphism in $\mathcal{C}$, minimal with respect to $\hat{e} S \neq\{0\}$, where $\hat{e}=\alpha(e, e)^{-1} e$, induces a bijection between the set of isomorphism 
classes of simple $k_{\alpha} \mathcal{C}$-modules and the set of isomorphism classes of pairs $(e, T)$ consisting of an idempotent endomorphism e in $\mathcal{C}$ and a simple $k_{\alpha} G_{e}$-module $T$.

Theorem 1.1 is, of course, simply the special case of Theorem 1.2 applied to $\alpha=$ 1 , but in order to separate the rather tedious technicalities related to the 2-cocycle $\alpha$ from the structural arguments underlying the proof, we first prove Theorem 1.1 and then adapt this proof to the twisted case.

Remark 1.3. Certain combinatorially defined algebras, such as Brauer algebras, Temperley-Lieb algebras, their cyclotomic analogues, Jones algebras and partition algebras can be interpreted as twisted monoid algebras; see Wilcox [24. In conjunction with Theorem 1.2 this yields alternative proofs for the parametrisations of simple modules for these algebras, without referring to their cellularity. Denote by $B_{n}(\delta)$ the Brauer algebra over a field $k$ with parameter $\delta \in k^{\times}$, where $n \geq 2$. By a result of Graham and Lehrer [7, (4.17)], the isomorphism classes of simple $B_{n}(\delta)$-modules are parametrised by pairs $(t, \lambda)$, where $t \geq 0$ is an integer such that $n-t$ is nonnegative and even, and where $\lambda$ is a partition of $t$ (a $p$-regular partition if the characteristic $p$ of $k$ is positive). This is related to Theorem 1.2 as follows. Any integer $t$ as above yields an idempotent $e_{t}$ in the semigroup $B R_{n}$ of diagrams which form a basis of $B_{n}(\delta)$; the idempotent $e_{t}$ corresponds to a diagram with $t$ strictly vertical strands and with horizontal strands connecting the remaining nodes (see [24, §8] for more details). This yields a set of representatives of the isomorphism classes of idempotents in the semigroup $B R_{n}$. Moreover, the group $G_{e_{t}}$ corresponding to the idempotent $e_{t}$ is isomorphic to the symmetric group $S_{t}$. The 2-cocycle arising in the Brauer algebra yields the trivial class upon restriction to $S_{t}$. The second component $\lambda$ corresponds to an isomorphism class of simple $k S_{t}$-modules. Thus the parametrisation from Theorem 1.2 yields that in [7, (4.17)]. The Temperley-Lieb algebra $T L_{n}(\delta)$ is the subalgebra of $B_{n}(\delta)$ spanned by the submonoid $T L_{n}$ of $B R_{n}$ of diagrams in $B R_{n}$ which do not have any intersecting strands. The semigroup $T L_{n}$ contains the idempotents $e_{t}$, and the coresponding groups $G_{e_{t}}$ are trivial. Thus, by Theorem 1.2, the isomorphism classes of simple $T L_{n}(\delta)$-modules are parametrised by the set of integers $t \geq 0$ such that $n-t \geq 0$ is even; this is [7, (6.8)]. Similarly, one can use Theorem 1.2 to obtain the parametrisation of the isomorphism classes of simple modules over the Jones algebra in 7 , (6.17)] and over partition algebras in [25, 4.11]. The same approach should also work for the cyclotomic Brauer algebras [11, 20] and cyclotomic Temperley-Lieb algebras [19] with additional parameter $m>0$. These algebras have idempotents as above, with associated groups of invertible elements isomorphic to $\mathbb{Z} / m Z \imath S_{t}$ and direct products of copies of $\mathbb{Z} / m \mathbb{Z}$, respectively. Therefore, Theorem 1.2 should yield again parametrisations, as in [19, 5.4], [20, 5.9], and [11, Prop. 43], of the isomorphism classes of simple modules for these algebras.

Remark 1.4. Twisted fusion category algebras arise in the context of reformulations of Alperin's weight conjecture in [12, [13. Theorem 1.2 suggests how to extend the notion of weights to arbitrary twisted finite category algebras: with the notation of Theorem 1.2 a weight of $k_{\alpha} \mathcal{C}$ is a pair $(e, T)$ consisting of an idempotent in $\mathcal{C}$ and a simple and projective $k_{\alpha} G_{e}$-module $T$. Denote by $w\left(k_{\alpha} \mathcal{C}\right)$ the number of isomorphism classes of weights of $k_{\alpha} \mathcal{C}$. The isomorphism classes of weights of $k_{\alpha} \mathcal{C}$ correspond to a subset of the isomorphism classes of simple $k_{\alpha} \mathcal{C}$-modules, through the correspondence in Theorem 1.2. Thus, if we choose an idempotent $c$ in $k_{\alpha} \mathcal{C}$ 
which annihilates exactly all simple modules which do not correspond to a weight, the resulting algebra $c \cdot k_{\alpha} \mathcal{C} \cdot c$ has exactly $w\left(k_{\alpha} \mathcal{C}\right)$ isomorphism classes of simple modules. This algebra has been considered in the context of finite $E I$-categories and fusion systems in 12, where it is shown to be quasi-hereditary (cf. [12, 2.4, 4.5]). Combining Theorem 1.2 with Alperin's weight conjecture yields a conjecture on the number of isomorphism classes of simple modules of twisted finite category algebras over algebraically closed fields of positive characteristic.

Remark 1.5. Let $\mathcal{C}$ be a finite category and $k$ a complete local Noetherian commutative ring with a residue field of prime characteristic $p$. Let $S$ be a simple $k \mathcal{C}$-module and $e$ a minimal idempotent in $\operatorname{Mor}(\mathcal{C})$ such that $e S \neq\{0\}$. Let $(Q, V)$ be a vertex-source pair for the simple $k G_{e}$-module $e S$; that is, $Q$ is a minimal subgroup of $G_{e}$ such that $e S$ is relatively $k Q$-projective and $V$ is an indecomposable $k Q$-module such that $e S$ is isomorphic to a direct summand of $\operatorname{Ind}_{Q}^{G_{e}}(V)$. By Green's work in [9], the group $Q$ is a $p$-group and the pair $(Q, V)$ is uniquely determined up to conjugation in $G_{e}$; see for instance [2, 3.10] or [22, Chapter 3] for expositions of these concepts. It is tempting to call $(Q, V)$ a vertex-source pair of the simple $k \mathcal{C}$-module $S$. In the context of finite group algebras, a vertex-source pair can be associated with any indecomposable module. This raises the question how to generalise vertices and sources to arbitrary indecomposable modules over the category algebra $k \mathcal{C}$. For finite $E I$-categories, Fei Xu has developed in [26] a notion of vertices and sources, where the vertices are certain full subcategories.

\section{Green Relations for CAtegories}

The results in this section are translations of basic results from semigroups to categories. For the most part the proofs, included here for the convenience of the reader, are straightforward adaptations of the proofs in the semigroup case; see, for instance, the exposition in [18, Appendix A]. Let $\mathcal{C}$ be a small category, with morphism set denoted $\operatorname{Mor}(\mathcal{C})$. For subsets $M, M^{\prime}$ of $\operatorname{Mor}(\mathcal{C})$ we denote by $M \circ M^{\prime}$ the (possibly empty) subset of $\operatorname{Mor}(\mathcal{C})$ consisting of all morphisms of the form $s \circ s^{\prime}$, where $s \in M$ and $s^{\prime} \in M^{\prime}$ such that this composition is defined. If $M$ consists of a single morphism $s$ we also write $s \circ M^{\prime}$ instead of $M \circ M^{\prime}$; similarly for $M^{\prime}$. We use the obvious generalisation of this notation for the composition of more than two subsets of $\operatorname{Mor}(\mathcal{C})$. Following the standard notation of the Green relations for semigroups [8], for any $s, t \in \operatorname{Mor}(\mathcal{C})$ we write $s \mathscr{L} t$ if $\operatorname{Mor}(\mathcal{C}) \circ s=\operatorname{Mor}(\mathcal{C}) \circ t$, we write $s \mathscr{R} t$ if $s \circ \operatorname{Mor}(\mathcal{C})=t \circ \operatorname{Mor}(\mathcal{C})$, and we write $s \mathscr{J} t$ if $\operatorname{Mor}(\mathcal{C}) \circ s \circ \operatorname{Mor}(\mathcal{C})=$ $\operatorname{Mor}(\mathcal{C}) \circ t \circ \operatorname{Mor}(\mathcal{C})$. A morphism $s \in \operatorname{Hom}_{\mathcal{C}}(X, Y)$ is called split if there is a morphism $t \in \operatorname{Hom}_{\mathcal{C}}(Y, X)$ satisfying $s=s \circ t \circ s$. (In the context of semigroups, this property is called regular or von Neumann regular.) The morphism $t$ need not be unique, and also $t$ need not be split itself, but one can always choose $t$ to be split: just replace $t$ by $u=t \circ s \circ t$; an easy calculation shows that we still have $s=$ $s \circ u \circ s$ and in addition $u=u \circ s \circ u$.

Lemma 2.1. Let $\mathcal{C}$ be a finite category and $X, Y$ objects in $\mathcal{C}$. Two idempotent endomorphisms $e \in \operatorname{End}_{\mathcal{C}}(X)$ and $f \in \operatorname{End}_{\mathcal{C}}(Y)$ are isomorphic if and only if e $\mathscr{J} f$.

Proof. Suppose that $e$ and $f$ are isomorphic. That is, there are morphisms $s \in$ $f \circ \operatorname{Hom}_{\mathcal{C}}(X, Y) \circ e$ and $t \in e \circ \operatorname{Hom}_{\mathcal{C}}(Y, X) \circ f$ such that $t \circ s=e$ and $s \circ t=f$. Thus $e=t \circ f \circ s$; hence $\operatorname{Mor}(\mathcal{C}) \circ e \circ \operatorname{Mor}(\mathcal{C}) \subseteq \operatorname{Mor}(\mathcal{C}) \circ f \circ \operatorname{Mor}(\mathcal{C})$. Exchanging the roles of $e$ and $f$ yields the equality of these two sets, whence $e \mathscr{J} f$. Conversely, 
suppose that $\operatorname{Mor}(\mathcal{C}) \circ e \circ \operatorname{Mor}(\mathcal{C})=\operatorname{Mor}(\mathcal{C}) \circ f \circ \operatorname{Mor}(\mathcal{C})$. Since $e$ is an idempotent, this equality implies that $e=t \circ s$ for some $s \in f \circ \operatorname{Hom}_{\mathcal{C}}(X, Y) \circ e$ and some $t \in$ $e \circ \operatorname{Hom}_{\mathcal{C}}(Y, X) \circ f$. Similarly, $f=s^{\prime} \circ t^{\prime}$ for some $s^{\prime} \in f \circ \operatorname{Hom}_{\mathcal{C}}(X, Y) \circ e$ and some $t^{\prime} \in e \circ \operatorname{Hom}_{\mathcal{C}}(Y, X) \circ f$. Thus $t^{\prime} \circ s$ and $t \circ s^{\prime}$ are both in $e \circ \operatorname{End}_{\mathcal{C}}(X) \circ e$ and we have $\left(t \circ s^{\prime}\right) \circ\left(t^{\prime} \circ s\right)=t \circ\left(s^{\prime} \circ t^{\prime}\right) \circ s=t \circ f \circ s=t \circ s=e$. Thus $t \circ s^{\prime}$ is left

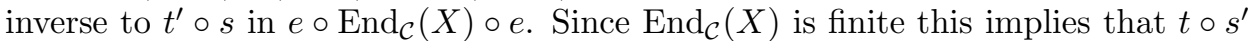
is also right inverse to $t^{\prime} \circ s$; thus $t^{\prime} \circ s \circ t \circ s^{\prime}=e$. Setting $u=s^{\prime} \circ t^{\prime} \circ s$ and $v=$ $t \circ s^{\prime} \circ t^{\prime}$, a straightforward verification yields $v \circ u=e$ and $u \circ v=f$, whence $e, f$ are isomorphic.

Lemma 2.2. Let $\mathcal{C}$ be a finite category and let $s, t$ be morphisms in $\mathcal{C}$ such that $t \circ s$ is defined.

(i) We have $s \mathscr{J}(t \circ s)$ if and only if $s \mathscr{L}(t \circ s)$.

(ii) We have $t \mathscr{J}(t \circ s)$ if and only if $t \mathscr{R}(t \circ s)$.

Proof. Suppose that $s \mathscr{J}(t \circ s)$. Then $s=u \circ t \circ s \circ v$ for some morphisms $u, v$. Note that $u \circ t$ and $v$ are endomorphisms of the target and source of $s$, respectively. Iterating the previous equation yields $s=(u \circ t)^{n} \circ s \circ v^{n}$ for all positive integers $n$. Since $\operatorname{Mor}(\mathcal{C})$ is finite, there is a positive integer $n$ such that $(u \circ t)^{n}$ is an idempotent endomorphism. Thus $s=(u \circ t)^{n} \circ(u \circ t)^{n} \circ s \circ v^{n}=(u \circ t)^{n} \circ s$, which is contained in $\operatorname{Mor}(\mathcal{C}) \circ t \circ s$, whence $s \mathscr{L}(t \circ s)$. The converse implication in (i) is obvious. Statement (ii) is proved analogously.

A semigroup satisfying the conclusions of Lemma 2.2 is called stable, but we do not use this terminology here in order to avoid confusion with the notion of stable module categories.

Lemma 2.3. Let $\mathcal{C}$ be a finite category and let $s, t \in \operatorname{Mor}(\mathcal{C})$ such that $s \mathscr{J} t$. There is a morphism $u \in \operatorname{Mor}(\mathcal{C})$ such that the composition $u \circ s$ is defined and such that we have $s \mathscr{L}(u \circ s)$ and $(u \circ s) \mathscr{R} t$.

Proof. Since $s \mathscr{J} t$ there are morphisms $u, v$ such that $t=u \circ s \circ v$. Since $s \mathscr{J} t$ we have $s \mathscr{J}(u \circ s)$; hence $s \mathscr{L}(u \circ s)$ by Lemma2.2. Similarly, we have $(u \circ s) \mathscr{J}(u \circ s \circ v)$; hence $(u \circ s) \mathscr{R}(u \circ s \circ v)$.

Lemma 2.4. Let $\mathcal{C}$ be a small category and let $s, t$ be morphisms in $\mathcal{C}$. Suppose that $s \mathscr{R} t$ or $s \mathscr{L} t$. Then $s$ is split if and only if $t$ is split.

Proof. Suppose that $s$ is split; that is, $s=s \circ r \circ s$ for some morphism $r$. If $s \mathscr{L} t$ there are morphisms $u, v$ such that $s=u \circ t$ and $t=v \circ s$. Then $t=v \circ s=$ $v \circ s \circ r \circ s=t \circ r \circ s=t \circ r \circ u \circ t$, and hence $t$ is split. A similar argument shows that if $s \mathscr{R} t$, then $t$ is split. Exchanging the roles of $s$ and $t$ concludes the proof.

Lemma 2.5. Let $\mathcal{C}$ be a finite category and let $J$ be a $\mathscr{J}$-equivalence class of morphisms in $\mathcal{C}$. The following statements are equivalent.

(i) $J$ contains an idempotent endomorphism of some object in $\mathcal{C}$.

(ii) $J$ contains a split morphism in $\mathcal{C}$.

(iii) Every morphism in $J$ is split.

(iv) $J \circ J \cap J$ is nonempty. 
Proof. The implication (i) $\Rightarrow$ (ii) is clear since every idempotent is split. In order to show (ii) $\Rightarrow$ (iii), let $s, t \in J$ and assume that $s$ is split. Let $u, v \in \operatorname{Mor}(\mathcal{C})$ be such that $t=u \circ s \circ v$. By Lemma 2.3 we have $s \mathscr{L}(u \circ s) \mathscr{R} t$ and by Lemma 2.4 both $u \circ s$ and $t$ are split. In order to show the implication (iii) $\Rightarrow$ (iv) let $s \in J$ such that $s \circ t \circ s=s$ for some $t \in \operatorname{Mor}(\mathcal{C})$. Then $(s \circ t) \mathscr{J} s$; hence $s \circ t, s \in J$, and hence $s=s \circ t \circ s \in J \circ J \cap J$. For the implication (iv) $\Rightarrow$ (i) let $s, t \in J$ such that $s \circ t$ is defined and in $J$. Then $s \mathscr{J}(s \circ t)$ and $t \mathscr{J}(s \circ t)$. By Lemma 2.2 we have $s \mathscr{R}(s \circ t)$ and $t \mathscr{L}(s \circ t)$; hence there are two morphisms $u, v \in \operatorname{Mor}(\mathcal{C})$ such that $s=s \circ t \circ u$ and $t=v \circ s \circ t$. Note that $t \circ u \in J$. Then $t \circ u \circ t \circ u=$ $(v \circ s \circ t) \circ u \circ t \circ u=v \circ(s \circ t \circ u) \circ t \circ u=v \circ s \circ t \circ u=t \circ u$.

A $\mathscr{J}$-class $J$ in the morphism set $\operatorname{Mor}(\mathcal{C})$ of a finite category $\mathcal{C}$ is called split if $J$ satisfies the equivalent conditions of Lemma 2.5. For $e$ an idempotent endomorphism of an object $X$ in $\mathcal{C}$ we denote as before by $G_{e}$ the group of invertible elements of the monoid $e \circ \operatorname{End}_{\mathcal{C}}(X) \circ e$.

Lemma 2.6. Let $\mathcal{C}$ be a finite category, $X$ an object in $\mathcal{C}$ and $e$ an idempotent in $\operatorname{End}_{\mathcal{C}}(X)$. Denote by $J$ the $\mathscr{J}$-class of e in $\operatorname{Mor}(\mathcal{C})$. Then eoEnd $\operatorname{En}_{\mathcal{C}}(X) \circ e \cap J=G_{e}$ and the complement of $G_{e}$ in the monoid $e \circ \operatorname{End}_{\mathcal{C}}(X) \circ e$ is a two-sided ideal.

Proof. Let $s \in e \circ \operatorname{End}_{\mathcal{C}}(X) \circ e$. Then $s=e \circ s$, and hence, by Lemma 2.2, we have $e \mathscr{J} s$ if and only if $e \mathscr{R} s$. Since $s=e \circ s$, the latter is equivalent to $e=s \circ u$ for some morphism $u$ in $e \circ \operatorname{End}_{\mathcal{C}}(X) \circ e$, which is in turn equivalent to $s \in G_{e}$ as the monoid $e \circ \operatorname{End}_{\mathcal{C}}(X) \circ e$ is finite. The last statement is obvious.

The set of idempotents in the morphism set $\operatorname{Mor}(\mathcal{C})$ of a small category $\mathcal{C}$ is partially ordered via $e \leq f$ if $e, f$ are idempotent endomorphisms of the same object in $\mathcal{C}$ such that $e=e \circ f=f \circ e$. The set of $\mathscr{J}$-classes in $\operatorname{Mor}(\mathcal{C})$ is partially ordered via $J \leq J^{\prime}$ if $J, J^{\prime}$ are $\mathscr{J}$-classes such that for some (hence any) $s \in J$, $s^{\prime} \in J^{\prime}$ we have $\operatorname{Mor}(\mathcal{C}) \circ s \circ \operatorname{Mor}(\mathcal{C}) \subseteq \operatorname{Mor}(\mathcal{C}) \circ s^{\prime} \circ \operatorname{Mor}(\mathcal{C})$. If $J$ is the $\mathscr{J}$-class of a morphism $s$, then the set $\operatorname{Mor}(\mathcal{C}) \circ s \circ \operatorname{Mor}(\mathcal{C})$ is the disjoint union of all $\mathscr{J}$-classes $J^{\prime}$ such that $J^{\prime} \leq J$. The map sending an idempotent to its $\mathscr{J}$-class preserves these partial orders; more precisely:

Lemma 2.7. Let $\mathcal{C}$ be a finite category and let $J, J^{\prime}$ be split $\mathscr{J}$-classes in $\operatorname{Mor}(\mathcal{C})$.

(i) If $e \in J$ and $e^{\prime} \in J^{\prime}$ are idempotents such that $e \leq e^{\prime}$, then $J \leq J^{\prime}$.

(ii) If $J \leq J^{\prime}$, then for any idempotent $e^{\prime} \in J^{\prime}$ there is an idempotent $e \in J$ such that $e \leq e^{\prime}$.

Proof. If $e \leq e^{\prime}$, then $e=e \circ e^{\prime}$; hence $e \in \operatorname{Mor}(\mathcal{C}) \circ e^{\prime} \circ \operatorname{Mor}(\mathcal{C})$, which implies $J \leq$ $J^{\prime}$, whence (i). Suppose that $J \leq J^{\prime}$. Then there are idempotents $e \in J$ and $e^{\prime} \in$ $J^{\prime}$ such that $\operatorname{Mor}(\mathcal{C}) \circ e \circ \operatorname{Mor}(\mathcal{C}) \subseteq \operatorname{Mor}(\mathcal{C}) \circ e^{\prime} \circ \operatorname{Mor}(\mathcal{C})$. In particular, $e=u \circ e^{\prime} \circ v$ for some morphisms $u, v$. Since $e, e^{\prime}$ are idempotents we may choose $u, v$ such that $e \circ u=u=u \circ e^{\prime}$ and $e^{\prime} \circ v=v=v \circ e$. Then $v \circ u$ is defined and $v \circ u \circ v \circ u=$ $v \circ e \circ u=v \circ u$; hence $v \circ u$ is an idempotent isomorphic to $e$. Moreover, we have $e^{\prime} \circ v \circ u=v \circ u=v \circ u \circ e^{\prime}$; hence $v \circ u \leq e^{\prime}$, which proves (ii). 


\section{Proof of Theorem 1.1}

Let $\mathcal{C}$ be a finite category. For any morphism $s$ in $\operatorname{Mor}(\mathcal{C})$ we set

$$
I_{s}=\{t \in \operatorname{Mor}(\mathcal{C}) \mid s \notin \operatorname{Mor}(\mathcal{C}) \circ t \circ \operatorname{Mor}(\mathcal{C})\} .
$$

In other words, $I_{s}$ is the union of all $\mathscr{J}$-classes $J$ such that $J_{s} \not \leq J$, where $J_{s}$ is the $\mathscr{J}$-class containing $s$. In particular, $I_{s} \cap J_{s}=\emptyset$. The set $I_{s}$ is a two-sided ideal in $\operatorname{Mor}(\mathcal{C})$ and depends only on the $\mathscr{J}$-class of $s$. For any $k \mathcal{C}$-module $U$ we denote by $\operatorname{Ann}_{\mathcal{C}}(U)$ the set of all morphisms in $\mathcal{C}$ which annihilate $U$. The following proposition collects the main steps of the proof of Theorem 1.1

Proposition 3.1. Let $k$ be a commutative ring and $\mathcal{C}$ a finite category. Let $S$ be a simple $k \mathcal{C}$-module and $J$ a $\mathscr{J}$-class, minimal with respect to $J S \neq\{0\}$. The following hold.

(i) $J$ is split.

(ii) $J$ is unique.

(iii) If e $\in J$, then $\operatorname{Ann}_{\mathcal{C}}(S)=I_{e}$.

(iv) If $e \in J$ is an idempotent, then $e \circ \operatorname{End}_{\mathcal{C}}(X) \circ e=G_{e} \cup\left(e \circ I_{e} \circ e\right)$, and this union is disjoint.

Proof. (i) Let $s \in J$. As mentioned above, the set $\operatorname{Mor}(\mathcal{C}) \circ s \circ \operatorname{Mor}(\mathcal{C})$ is the disjoint union of all $\mathscr{J}$-classes $J^{\prime}$ such that $J^{\prime} \leq J$. Since any $\mathscr{J}$-class strictly smaller than $J$ annihilates $S$, we have $(k J) S=k(\operatorname{Mor}(\mathcal{C}) \circ s \circ \operatorname{Mor}(\mathcal{C})) S$. This is a nonzero submodule of the simple module $S$, hence equal to $S$. Applying this twice yields $S=k(J \circ J) S$; in particular, $J \circ J$ does not annihilate $S$. Since $J \circ J$ is a subset of $\operatorname{Mor}(\mathcal{C}) \circ s \circ \operatorname{Mor}(\mathcal{C})$, it follows that $J \circ J$ must contain an element of $J$ since otherwise $J \circ J$ would annihilate $S$. Thus, by Lemma 2.5 the $\mathscr{J}$-class $J$ is split.

(ii) Let $J^{\prime}$ be another $\mathscr{J}$-class such that $J^{\prime} S \neq\{0\}$. Then $\left(k J^{\prime}\right) S=k\left(J^{\prime} \circ J\right) S \neq$ $\{0\}$. All elements in $J^{\prime} \circ J$ belong to $\mathscr{J}$-classes contained in $J$. Thus $J^{\prime} \circ J \cap J \neq$ $\emptyset$, which forces $J \leq J^{\prime}$.

(iii) Let $s \in \operatorname{Ann}_{\mathcal{C}}(S)$. Since $s$ annihilates $S$, every element of $\operatorname{Mor}(\mathcal{C}) \circ s \circ \operatorname{Mor}(\mathcal{C})$ annihilates $S$. Since $J$ does not annihilate $S$, we have $J \nsubseteq \operatorname{Mor}(\mathcal{C}) \circ s \circ \operatorname{Mor}(\mathcal{C})$ and so $s \in I_{e}$. Conversely, if $s \in I_{e}$, then $e \notin \operatorname{Mor}(\mathcal{C}) \circ s \circ \operatorname{Mor}(\mathcal{C})$. Hence no $\mathscr{J}$-class $J^{\prime}$ in $\operatorname{Mor}(\mathcal{C}) \circ s \circ \operatorname{Mor}(\mathcal{C})$ satisfies $J \leq J^{\prime}$. Since, by (ii), $J$ is the unique minimal $\mathscr{J}$-class which does not annihilate $S$, it follows that $\operatorname{Mor}(\mathcal{C}) \circ s \circ \operatorname{Mor}(\mathcal{C})$ annihilates $S$, whence $s \in \operatorname{Ann}_{\mathcal{C}}(S)$.

(iv) As before we denote by $J_{s}$ the $\mathscr{J}$-class containing a morphism $s$. Let $s \in$ $e \circ \operatorname{End}_{\mathcal{C}}(X) \circ e$. If $s \in J$, then $s \in G_{e}$ by Lemma 2.6. Suppose $s \notin J$. Then $J_{s}<$ $J_{e}=J$; hence $s$ annihilates $S$, by the minimality of $J$ subject to not annihilating $S$. It follows that $s \in \operatorname{Ann}_{\mathcal{C}}(S)=I_{e}$, where the last equality uses (iii). Since $e$ is an idempotent, this implies that $s \in e \circ I_{e} \circ e$.

Proof of Theorem 1.1. Let $S$ be a simple $k \mathcal{C}$-module. Let $e$ be a minimal idempotent endomorphism of an object $X$ in $\mathcal{C}$ such that $e S \neq\{0\}$. By [10, 6.2], $e S$ is a simple $k\left(e \circ \operatorname{End}_{\mathcal{C}}(X) \circ e\right)$-module. By Lemma 2.7, the $\mathscr{J}$-class $J$ of $e$ is minimal such that $J S \neq\{0\}$. Thus $e S$ is annihilated by $e \circ I_{e} \circ e$, and hence, $e S$ remains simple as a $k G_{e}$-module. Again by [10, 6.2], the module $S$ is, up to isomorphism, determined by the isomorphism class of the pair $(e, e S)$, and hence the map sending $S$ to $(e, e S)$ becomes injective on isomorphism classes. It also becomes surjective 
since any simple $k G_{e}$-module $T$ can be viewed as a simple $k\left(e \circ \operatorname{End}_{\mathcal{C}}(X) \circ e\right)$-module in such a way that the complement of $G_{e}$ in $e \circ \operatorname{End}_{\mathcal{C}}(X) \circ e$ annihilates $T$.

\section{Green Relations For extensions of CATEGories}

Let $\mathcal{C}$ be a finite category and $A$ an abelian group, written multiplicatively. Let $\alpha$ be a 2-cocycle of $\mathcal{C}$ with coefficients in $A$. That is, $\alpha$ is a map from pairs of composable morphisms in $\mathcal{C}$ to $A$ satisfying the 2-cocycle identity. More precisely, if $s, t$ are morphisms in $\mathcal{C}$ such that $t \circ s$ is defined, then $\alpha(t, s)$ is an element in $A$, with the property that if $t, s, u$ are morphisms in $\mathcal{C}$ such that $t \circ s$ and $u \circ t$ are defined, then $\alpha(u \circ t, s) \alpha(u, t)=\alpha(u, t \circ s) \alpha(t, s)$. The extension category of $\mathcal{C}$ by $A$ associated with $\alpha$ is the category $\hat{\mathcal{C}}$ with object set $\operatorname{Ob}(\hat{\mathcal{C}})=\operatorname{Ob}(\mathcal{C})$ and morphism set $\operatorname{Mor}(\hat{\mathcal{C}})=\operatorname{Mor}(\mathcal{C}) \times A$, such that the composition in $\hat{\mathcal{C}}$ is defined by $(t, b) \circ(s, a)=(t \circ s, b a \alpha(t, s))$, for any two morphisms $s, t$ in $\mathcal{C}$ for which $t \circ s$ is defined and any $a, b \in A$. There is a canonical functor $\hat{\mathcal{C}} \rightarrow \mathcal{C}$ which is the identity on objects and which sends a morphism $(s, a)$ in $\hat{\mathcal{C}}$ to the morphism $s$ in $\mathcal{C}$. The isomorphism class of the extension $\hat{\mathcal{C}}$ of $\mathcal{C}$ by $A$ depends only on the class of $\alpha$ in $H^{2}(\mathcal{C} ; A)$. See [1, or also 23 , for an introductory survey on extensions and cohomology of small categories, as well as further references. We will use in the sequel the elementary fact that if $e, f$ are idempotent endomorphisms of objects $X$, $Y$, respectively, in $\mathcal{C}$, then $e, f$ are isomorphic if and only if there are morphisms $s \in \operatorname{Hom}_{\mathcal{C}}(X, Y)$ and $t \in \operatorname{Hom}_{\mathcal{C}}(Y, X)$ satisfying $e=t \circ s$ and $f=s \circ t$. Indeed, if there are such morphisms $s, t$, then the morphisms $s^{\prime}=f \circ s \circ e$ and $t^{\prime}=e \circ t \circ f$ are easily seen to still satisfy $t^{\prime} \circ s^{\prime}=e$ and $s^{\prime} \circ t^{\prime}=f$; hence $e$ and $f$ are isomorphic. The converse is trivial. The following observation shows that equivalence classes of Green relations in $\hat{\mathcal{C}}$ are precisely the inverse images of Green relations in $\mathcal{C}$. We keep the notation above.

Lemma 4.1. Let $s, t$ be morphisms in $\mathcal{C}$ and let $a, b \in A$.

(i) We have $s \mathscr{L} t$ if and only if $(s, a) \mathscr{L}(t, b)$.

(ii) We have $s \mathscr{R} t$ if and only if $(s, a) \mathscr{R}(t, b)$.

(iii) We have $s \mathscr{J} t$ if and only if $(s, a) \mathscr{J}(t, b)$.

(iv) The morphism $s$ is split if and only if $(s, a)$ is split.

Proof. Suppose that $s \mathscr{L} t$. Then there is a morphism $u$ such that $s=u \circ t$. A short calculation shows that $\left(u, a \alpha(u, t)^{-1} b^{-1}\right) \circ(t, b)=(s, a)$. Exchanging the roles of $s$ and $t$ yields that $(s, a) \mathscr{L}(t, b)$. The converse implication in (i) is trivial. The statements (ii) and (iii) are proved similarly. For (iv), suppose that $s=s \circ u \circ s$ for some morphism $u$ in $\mathcal{C}$. Setting $b=a^{-1} \alpha(s, u) \alpha(s \circ u, s)^{-1}$ one checks that $(s, a)=$ $(s, a) \circ(u, b) \circ(s, a)$; hence $(s, a)$ is split. The converse in (iv) is clear.

Note that Lemma 4.1 implies also that the Green relations in $\hat{\mathcal{C}}$ satisfy the conclusion of Lemma 2.2 (that is, $\hat{\mathcal{C}}$ is 'stable' in the semigroup sense, although $\hat{\mathcal{C}}$ need no longer be finite because $A$ need not be finite).

Lemma 4.2. Let $X, Y$ be objects in $\mathcal{C}$, let $e \in \operatorname{End}_{\mathcal{C}}(X)$ and $f \in \operatorname{End}_{\mathcal{C}}(Y)$ be idempotents and let $s \in f \circ \operatorname{Hom}_{\mathcal{C}}(X, Y) \circ e$. We have $\alpha(s, e)=\alpha(e, e)$ and $\alpha(f, s)=$ $\alpha(f, f)$. 
Proof. The 2-cocycle identity applied to $s, e, e$ yields $\alpha(s \circ e, e) \alpha(s, e)=\alpha(s, e \circ$ $e) \alpha(e, e)$. Since $s \circ e=s$ and $e \circ e=e$, the first equality follows from cancelling $\alpha(s, e)$ on both sides. The second equality follows similarly from the 2-cocycle identity applied to $f, f, s$.

Lemma 4.3. (i) The canonical functor $\hat{\mathcal{C}} \rightarrow \mathcal{C}$ induces a bijection between the set of idempotents in $\hat{\mathcal{C}}$ and the set of idempotents in $\mathcal{C}$. The inverse of this bijection sends an idempotent $e$ in $\mathcal{C}$ to the idempotent $\left(e, \alpha(e, e)^{-1}\right)$ in $\hat{\mathcal{C}}$.

(ii) For any two idempotents $e, f$ in $\mathcal{C}$, we have $e \leq f$ if and only if $\left(e, \alpha(e, e)^{-1}\right) \leq$ $\left(f, \alpha(f, f)^{-1}\right)$.

(iii) For any two idempotents $e, f$ in $\mathcal{C}$, we have $e \cong f$ if and only if $\left(e, \alpha(e, e)^{-1}\right) \cong$ $\left(f, \alpha(f, f)^{-1}\right)$.

Proof. Let $e, f$ be idempotent endomorphisms of objects $X, Y$, respectively, in $\mathcal{C}$. Let $a \in A$. Since $(e, a) \circ(e, a)=\left(e, a^{2} \alpha(e, e)\right)$, the unique idempotent in the inverse image of $e$ in the morphism set of $\hat{\mathcal{C}}$ is $\left(e, \alpha(e, e)^{-1}\right)$, whence (i). Suppose that $e \leq f$; that is, $X=Y$ and $e \circ f=e=f \circ e$. Using the equality $\alpha(e, f)=\alpha(f, f)$ from Lemma 4.2 we get that $\left(e, \alpha(e, e)^{-1}\right) \circ\left(f, \alpha(f, f)^{-1}\right)=\left(e, \alpha(e, e)^{-1}\right)$. A similar $\operatorname{argument}$ yields $\left(f, \alpha(f, f)^{-1}\right) \circ\left(e, \alpha(e, e)^{-1}\right)=\left(e, \alpha(e, e)^{-1}\right)$; hence $\left(e, \alpha(e, e)^{-1}\right) \leq$ $\left(f, \alpha(f, f)^{-1}\right)$. The converse in (ii) is obvious. Suppose next that $e \cong f$. Let $s \in$ $f \circ \operatorname{Hom}_{\mathcal{C}}(X, Y) \circ e$ and $t \in e \circ \operatorname{Hom}_{\mathcal{C}}(Y, X) \circ f$ such that $t \circ s=e$ and $s \circ t=f$. Set $b=\alpha(s, t)^{-1} \alpha(f, f)^{-1}$. Then $(s, 1) \circ(t, b)=(f, b \alpha(s, t))=\left(f, \alpha(f, f)^{-1}\right)$. The 2 -cocycle identity applied to $s, t, s$ yields $\alpha(s, t \circ s) \alpha(t, s)=\alpha(s \circ t, s) \alpha(s, t)$. Since $t \circ s=e$ we have $\alpha(s, t \circ s)=\alpha(s, e)=\alpha(e, e)$, where the last equality uses Lemma 4.2. Similarly, $\alpha(s \circ t, s)=\alpha(f, f)$. Thus $\alpha(e, e) \alpha(t, s)=\alpha(f, f) \alpha(s, t)$. This equality implies that $(t, b) \circ(s, 1)=\left(e, \alpha(e, e)^{-1}\right)$. This shows that $\left(e, \alpha(e, e)^{-1}\right)$ and $\left(f, \alpha(f, f)^{-1}\right)$ are isomorphic. The converse in (iii) is obvious.

\section{Proof of Theorem 1.2}

Let $\mathcal{C}$ be a finite category, $k$ a commutative ring and $\alpha$ a 2-cocycle of $\mathcal{C}$ with coefficients in $k^{\times}$. Denote by $k_{\alpha} \mathcal{C}$ the corresponding twisted category algebra of $\mathcal{C}$ over $k$ and by $\hat{\mathcal{C}}$ the corresponding extension category of $\mathcal{C}$ by $k^{\times}$. The map sending a morphism $(s, \lambda)$ in $\hat{\mathcal{C}}$ to $\lambda s$ induces a surjective algebra homomorphism $k \hat{\mathcal{C}} \rightarrow k_{\alpha} \mathcal{C}$. For any subset $M$ of $\operatorname{Mor}(\mathcal{C})$ denote by $k_{\alpha} M$ the $k$-submodule spanned by $M$ in $k_{\alpha} \mathcal{C}$. For $M, N$ two subsets of $\operatorname{Mor}(\mathcal{C})$ we have $k_{\alpha} M \cdot k_{\alpha} N=k_{\alpha}(M \circ N)$. In particular, if $M$ is a 2-sided ideal in $\operatorname{Mor}(\mathcal{C})$, then $k_{\alpha} M$ is a 2-sided ideal in the algebra $k_{\alpha} \mathcal{C}$. As before, for any morphism $s$ in $\mathcal{C}$ we denote by $I_{s}$ the set of all morphisms $t$ such that $s \notin \operatorname{Mor}(\mathcal{C}) \circ t \circ \operatorname{Mor}(\mathcal{C})$ and for any $k_{\alpha} \mathcal{C}$-module $U$ we denote by $\operatorname{Ann}_{\mathcal{C}}(U)$ the set of all morphisms in $\mathcal{C}$ which annihilate $U$. By the previous remarks, $\operatorname{Ann}_{\mathcal{C}}(U)$ is a 2 -sided ideal in $\operatorname{Mor}(\mathcal{C})$. We keep the notation above. Proposition 3.1 carries over to twisted category algebras:

Proposition 5.1. Let $S$ be a simple $k_{\alpha} \mathcal{C}$-module and $J$ a $\mathscr{J}$-class, minimal with respect to $J S \neq\{0\}$. The following hold.

(i) $J$ is split.

(ii) $J$ is unique.

(iii) If $e \in J$, then $\operatorname{Ann}_{\mathcal{C}}(S)=I_{e}$. 
(iv) If $e \in J$ is an idempotent, then $e \circ \operatorname{End}_{\mathcal{C}}(X) \circ e=G_{e} \cup\left(e \circ I_{e} \circ e\right)$, and this union is disjoint.

Proof. Using the preceding remarks one sees that the proof of Proposition 3.1 carries over without any change. Alternatively, one can prove this by interpreting $S$ as a simple module for the category algebra $k \hat{\mathcal{C}}$ through the canonical homomorphism $k \hat{\mathcal{C}} \rightarrow k_{\alpha} \mathcal{C}$, where $\hat{\mathcal{C}}$ is the extension of $\mathcal{C}$ by $k^{\times}$corresponding to $\alpha$. Although $\hat{\mathcal{C}}$ need not be finite, the conclusions of the results from $\$ 2$ needed for the proof of Proposition 3.1 are easily seen to hold for $\hat{\mathcal{C}}$ instead of $\mathcal{C}$.

We need to verify that there is a well-defined notion of isomorphism classes of pairs $(e, \hat{e} S)$ as in the statement of Theorem 1.2. More precisely, we will show in the following two propositions that isomorphic idempotents $e, f$ in $\mathcal{C}$ give rise to an isomorphism of twisted group algebras $k_{\alpha} G_{e} \cong k_{\alpha} G_{f}$ which is uniquely determined up to an inner algebra automorphism.

Proposition 5.2. Let e, $f$ be idempotent endomorphisms of objects $X, Y$, respectively, in $\mathcal{C}$, let $s \in f \circ \operatorname{Hom}_{\mathcal{C}}(X, Y) \circ e$ and $t \in e \circ \operatorname{Hom}_{\mathcal{C}}(Y, X) \circ f$ satisfying $t \circ s=$ $e$ and $s \circ t=f$. For $x \in G_{e}$, set $\beta(x)=\alpha(x, t) \alpha(s, x \circ t) \alpha(e, e)^{-1} \alpha(t, s)^{-1}$. The map sending $x \in G_{e}$ to $\beta(x)(s \circ x \circ t)$ induces a $k$-algebra isomorphism $k_{\alpha} G_{e} \cong$ $k_{\alpha} G_{f}$.

Proof. Since the map sending $x \in G_{e}$ to $s \circ x \circ t$ is a group isomorphism, $G_{e} \cong G_{f}$, the map sending $x \in G_{e}$ to $\beta(x)(s \circ x \circ t)$ induces a $k$-linear isomorphism, $k_{\alpha} G_{e} \cong$ $k_{\alpha} G_{f}$. It remains to show that this map is multiplicative. Let $x, y \in G_{e}$. The image of the product $x \cdot y=\alpha(x, y)(x \circ y)$ is equal to $\alpha(x, y) \beta(x \circ y)(s \circ x \circ y \circ t)$. The product of the images of $x, y$ in $k_{\alpha} G_{f}$ is equal to $\alpha(s \circ x \circ t, s \circ y \circ t) \beta(x) \beta(y)(s \circ x \circ y \circ t)$. Thus we need to show the equality

$$
\alpha(x, y) \beta(x \circ y)=\alpha(s \circ x \circ t, s \circ y \circ t) \beta(x) \beta(y) .
$$

We consider first the left side of this equation, which is equal to $\alpha(x, y) \alpha(x \circ$ $y, t) \alpha(s, x \circ y \circ t) \alpha(e, e)^{-1} \alpha(t, s)^{-1}$. The 2-cocycle identity applied to $x, y, t$ in the first two factors of this product yields the expression $\alpha(y, t) \alpha(x, y \circ t) \alpha(s, x \circ$ $y \circ t) \alpha(e, e)^{-1} \alpha(t, s)^{-1}$. The 2 -cocycle identity applied to $s, x, y \circ t$ in the second and third factor of this product yields the equality

$$
\alpha(x, y) \beta(x \circ y)=\alpha(y, t) \alpha(s, x) \alpha(s \circ x, y \circ t) \alpha(e, e)^{-1} \alpha(t, s)^{-1} .
$$

We need to show that this is equal to $\alpha(s \circ x \circ t, s \circ y \circ t) \beta(x) \beta(y)$. The 2-cocycle identity applied to $s \circ x \circ t, s, y \circ t$ yields $\alpha(s \circ x \circ t, s \circ y \circ t)=\alpha(s \circ x, y \circ t) \alpha(s \circ$ $x \circ t, s) \alpha(s, y \circ t)^{-1}$. The 2-cocycle identity applied to $s \circ x, t, s$ in the middle term of this expression yields $\alpha(s \circ x \circ t, s \circ y \circ t)=\alpha(s \circ x, y \circ t) \alpha(s \circ x, t \circ s) \alpha(t, s) \alpha(s \circ$ $x, t)^{-1} \alpha(s, y \circ t)^{-1}$. Using $t \circ s=e$ and $\alpha(s \circ x, e)=\alpha(e, e)$ from Lemma 4.2 yields $\alpha(s \circ x \circ t, s \circ y \circ t)=\alpha(s \circ x, y \circ t) \alpha(e, e) \alpha(t, s) \alpha(s \circ x, t)^{-1} \alpha(s, y \circ t)^{-1}$. Multiplying this equation by $\beta(x) \beta(y)$ yields $\alpha(s \circ x \circ t, s \circ y \circ t) \beta(x) \beta(y)=\alpha(s \circ x, y \circ t) \alpha(s \circ$ $x, t)^{-1} \alpha(x, t) \alpha(s, x \circ t) \alpha(e, e)^{-1} \alpha(t, s)^{-1} \alpha(y, t)$. The 2-cocycle identity applied to $s, x, t$ in the second, third and fourth factors shows that this is indeed equal to $\alpha(x, y) \beta(x \circ y)$, as required.

Lemma 5.3. Let e, $f$ be idempotent endomorphisms of objects $X, Y$, respectively, in $\mathcal{C}$, let $s \in f \circ \operatorname{Hom}_{\mathcal{C}}(X, Y) \circ e$ and $t \in e \circ \operatorname{Hom}_{\mathcal{C}}(Y, X) \circ f$ satisfying $t \circ s=e$ and $s \circ t=f$. We have $\alpha(t, s) \alpha(e, e)=\alpha(s, t) \alpha(f, f)$. In particular, if $e=f$, then $\alpha(t, s)=\alpha(s, t)$. 
Proof. This follows from Lemma 4.2 and the 2-cocycle identity applied to the morphisms $t, s$ and $t$.

Proposition 5.4. Let $e, f$ be idempotent endomorphisms of objects $X, Y$, respectively, in $\mathcal{C}$. Let $s, s^{\prime} \in f \circ \operatorname{Hom}_{\mathcal{C}}(X, Y) \circ e$ and $t, t^{\prime} \in e \circ \operatorname{Hom}_{\mathcal{C}}(Y, X) \circ f$ satisfying $t \circ s=e=t^{\prime} \circ s^{\prime}$ and $s \circ t=f=s^{\prime} \circ t^{\prime}$. For $x \in G_{e}$, set $\beta(x)=\alpha(x, t) \alpha(s, x \circ$ t) $\alpha(e, e)^{-1} \alpha(t, s)^{-1}$. For $y \in G_{f}$, set $\beta^{\prime}(y)=\alpha\left(y, s^{\prime}\right) \alpha\left(t^{\prime}, y \circ s^{\prime}\right) \alpha(f, f)^{-1} \alpha\left(s^{\prime}, t^{\prime}\right)^{-1}$. The map sending $x \in G_{e}$ to $\beta(x) \beta^{\prime}(s \circ x \circ t)\left(t^{\prime} \circ s \circ x \circ t \circ s^{\prime}\right)$ induces an inner automorphism of the $k$-algebra $k_{\alpha} G_{e}$.

Proof. We will show that the map sending $x \in G_{e}$ to $\beta(x) \beta^{\prime}(s \circ x \circ t)\left(t^{\prime} \circ s \circ x \circ t \circ s^{\prime}\right)$ coincides with conjugation by $a=t^{\prime} \circ s$ in $k_{\alpha} G_{e}$. Note that $t \circ s^{\prime}$ is the inverse of $t^{\prime} \circ s$ in the group $G_{e}$ but not necessarily in the algebra $k_{\alpha} G_{e}$. More precisely, since the unit element in $k_{\alpha} G_{e}$ is equal to $\alpha(e, e)^{-1} e$, the inverse of $a=t^{\prime} \circ s$ in $k_{\alpha} G_{e}$ is equal to $a^{-1}=\alpha(e, e)^{-1} \alpha\left(t \circ s^{\prime}, t^{\prime} \circ s\right)^{-1}\left(t \circ s^{\prime}\right)$. We have

$a \cdot x \cdot a^{-1}=\alpha(e, e)^{-1} \alpha\left(t \circ s^{\prime}, t^{\prime} \circ s\right)^{-1} \alpha\left(t^{\prime} \circ s, x\right) \alpha\left(t^{\prime} \circ s \circ x, t \circ s^{\prime}\right)\left(t^{\prime} \circ s \circ x \circ t \circ s^{\prime}\right)$.

Thus we need to show the equality

$$
\beta(x) \beta^{\prime}(s \circ x \circ t)=\alpha(e, e)^{-1} \alpha\left(t \circ s^{\prime}, t^{\prime} \circ s\right)^{-1} \alpha\left(t^{\prime} \circ s, x\right) \alpha\left(t^{\prime} \circ s \circ x, t \circ s^{\prime}\right) .
$$

Bringing all terms with an inverse sign to the other side and cancelling one factor $\alpha(e, e)$ shows that this equality is equivalent to the equality

$$
\begin{gathered}
\alpha(x, t) \alpha(s, x \circ t) \alpha\left(s \circ x \circ t, s^{\prime}\right) \alpha\left(t^{\prime}, s \circ x \circ t \circ s^{\prime}\right) \alpha\left(t^{\prime} \circ s, t \circ s^{\prime}\right) \\
=\alpha(t, s) \alpha(f, f) \alpha\left(s^{\prime}, t^{\prime}\right) \alpha\left(t^{\prime} \circ s, x\right) \alpha\left(t^{\prime} \circ s \circ x, t \circ s^{\prime}\right) .
\end{gathered}
$$

The 2-cocycle identity for $s, x \circ t, s^{\prime}$ applied to the second and third factors on the left side shows that the left side is equal to

$$
\alpha(x, t) \alpha\left(x \circ t, s^{\prime}\right) \alpha\left(s, x \circ t \circ s^{\prime}\right) \alpha\left(t^{\prime}, s \circ x \circ t \circ s^{\prime}\right) \alpha\left(t^{\prime} \circ s, t \circ s^{\prime}\right) .
$$

The 2-cocycle identity for $x, t, s^{\prime}$ applied to the first and second factors, and the 2-cocycle identity for $t^{\prime}, s, x \circ t \circ s^{\prime}$ applied to the third and fourth factors of this expression show that the left side above is equal to

$$
\alpha\left(t, s^{\prime}\right) \alpha\left(x, t \circ s^{\prime}\right) \alpha\left(t^{\prime}, s\right) \alpha\left(t^{\prime} \circ s, x \circ t \circ s^{\prime}\right) \alpha\left(t^{\prime} \circ s, t \circ s^{\prime}\right) .
$$

The 2-cocycle identity for $t^{\prime}, s, t \circ s^{\prime}$ applied to the third and fifth factors yields the expression

$$
\alpha\left(t, s^{\prime}\right) \alpha\left(x, t \circ s^{\prime}\right) \alpha\left(t^{\prime}, s^{\prime}\right) \alpha\left(s, t \circ s^{\prime}\right) \alpha\left(t^{\prime} \circ s, x \circ t \circ s^{\prime}\right) .
$$

The 2-cocycle identity for $s, t, s^{\prime}$ applied to the first and fourth factors (using $s \circ t=$ $f$ and $\alpha\left(f, s^{\prime}\right)=\alpha(f, f)$ from Lemma 4.2) yields

$$
\alpha(f, f) \alpha(s, t) \alpha\left(x, t \circ s^{\prime}\right) \alpha\left(t^{\prime}, s^{\prime}\right) \alpha\left(t^{\prime} \circ s, x \circ t \circ s^{\prime}\right) .
$$

The 2-cocycle identity for $t \circ s^{\prime}, x, t \circ s^{\prime}$ applied to the third and fifth factors yields the expression

$$
\alpha(f, f) \alpha(s, t) \alpha\left(t^{\prime} \circ s, x\right) \alpha\left(t^{\prime} \circ s \circ x, t \circ s^{\prime}\right) \alpha\left(t^{\prime}, s^{\prime}\right) .
$$

Using Lemma 5.3 twice shows that this is equal to the expression on the right side of the above equation. 
Proof of Theorem 1.2. The above results in this section imply that the proof of Theorem 1.1 carries over to twisted category algebras. Let $S$ be a simple $k_{\alpha} \mathcal{C}$ module and $e$ a minimal idempotent endomorphism of an object $X$ in $\mathcal{C}$ such that $\hat{e} S \neq\{0\}$, where $\hat{e}=\alpha(e, e)^{-1} e$. By [10, 6.2], $\hat{e} S$ is a simple $k_{\alpha}\left(e \circ \operatorname{End}_{\mathcal{C}} \circ e\right)$-module. By Lemma 2.7, the $\mathscr{J}$-class $J$ of $e$ is minimal such that $J S \neq\{0\}$. Thus $\hat{e} S$ is annihilated by $e \circ I_{e} \circ e$, and hence, $\hat{e} S$ remains simple as a $k_{\alpha} G_{e}$-module. By Propositions 5.2 and 5.4, we have a well-defined notion of isomorphism between pairs of the form $(e, \hat{e} S)$. Again by [10,6.2], the module $S$ is, up to isomorphism, determined by the isomorphism class of the pair $(e, \hat{e} S)$, and hence the map sending $S$ to $(e, \hat{e} S)$ becomes injective on isomorphism classes. It also becomes surjective since, by Proposition 5.1 any simple $k_{\alpha} G_{e}$-module $T$ can be viewed as a simple $k_{\alpha}\left(e \circ \operatorname{End}_{\mathcal{C}}(X) \circ e\right)$-module in such a way that the complement of $G_{e}$ in $e \circ \operatorname{End}_{\mathcal{C}}(X) \circ e$ annihilates $T$.

\section{REFERENCES}

[1] H.-J. Baues, G. Wirsching, Cohomology of small categories, J. Pure Appl. Algebra 38 (1985), 187-211. MR814176 (87g:18013)

[2] D. J. Benson, Representations and Cohomology, Vol. I, Cambridge Studies in Advanced Mathematics 30, Cambridge University Press, 1991. MR.1110581 (92m:20005)

[3] A. H. Clifford, Matrix representations of completely simple semigroups, Amer. J. Math. 64 (1942), 327-342. MR0006551 (4:4a)

[4] A. H. Clifford, Basic representations of completely simple semigroups, Amer. J. Math. 82 (1960), 430-434. MR0116062 (22:6857)

[5] T. tom Dieck, Transformation Groups, Walter de Gruyter, Berlin, New York, 1987. MR889050 (89c:57048)

[6] O. Ganyushkin, V. Mazorchuk and B. Steinberg, On the irreducible representations of a finite semigroup, Proc. Amer. Math. Soc. 137 (2009), 3585-3592. MR2529864 (2010h:20150)

[7] J. J. Graham and G. I. Lehrer, Cellular algebras, Invent. Math. 123 (1996), 1-34. MR.1376244 (97h:20016)

[8] J. A. Green, On the structure of semigroups, Ann. of Math. (2) 54 (1951), 163-172. MR0042380(13:100d)

[9] J. A. Green, On the indecomposable representations of a finite group, Math. Z. 70 (1959), 430-445. MR0131454 (24:A1304)

[10] J. A. Green, Polynomial representations of $\mathrm{GL}_{n}$, Lecture Notes in Math. 830, SpringerVerlag, Berlin, 1980. MR606556 (83j:20003)

[11] R. Häring-Oldenburg, Cyclotomic Birman-Murakami-Wenzl algebras, J. Pure Appl. Algebra 161 (2001), 113-144. MR.1834081 (2002c:20055)

[12] M. Linckelmann, Fusion category algebras. J. Algebra 277 (2004), 222-235. MR2059628 (2005a:20017)

[13] M. Linckelmann, Alperin's weight conjecture in terms of equivariant Bredon cohomology. Math. Z. 250 (2005), 495-513. MR.2179608 (2006i:20010)

[14] M. Linckelmann, On inverse categories and transfer in cohomology, Skye conference proceedings (2009), to appear.

[15] W. Lück, Transformation Groups and Algebraic K-Theory, Springer Lecture Notes Math. 1408 (1989). MR 1027600 (91g:57036)

[16] W. D. Munn, Matrix representations of semi-groups, Proc. Cambridge Philos. Soc. 53 (1957), 5-12. MR0082050 (18:489g)

[17] I. S. Ponizovskiǔ, On matrix representations of associative systems, Mat. Sb. N. S. 38 (1956), 241-260. MR0081292(18:378d)

[18] J. Rhodes and B. Steinberg, The q-Theory of Finite Semigroups, Springer Monographs in Mathematics (2009). MR2472427 (2010h:20132)

[19] H. Rui and Ch. Xi, The representation theory of cyclotomic Temperley-Lieb algebras, Comment. Math. Helvet. 79 (2004), 427-450. MR2059440 (2005b:16027)

[20] H. Rui and W. Yu, On the semi-simplicity of the cyclotomic Brauer algebras, J. Algebra 277 (2004), 187-221. MR2059627 (2005b:16057) 
[21] B. Steinberg, Möbius functions and semigroup representation theory II: Character formulas and multiplicities, Advances in Math. 217 (2008), 1521-1557. MR2382734 (2009e:20131)

[22] J. Thévenaz, G-Algebras and Modular Representation Theory, Oxford Science Publications, Clarendon Press, Oxford (1995). MR.1365077 (96j:20017)

[23] P. J. Webb, An introduction to the representations and cohomology of categories, in: Group Representation Theory, M. Geck, D. Testerman, J. Thévenaz (eds.), EPFL Press, Lausanne (2007), 149-173. MR2336640 (2008f:18013)

[24] S. Wilcox, Cellularity of diagram algebras as twisted semigroup algebras, J. Algebra 309 (2007), 10-31. MR2301230 (2008a:16041)

[25] Ch. Xi, Partition algebras are cellular, Compositio Mathematica 119 (1999), 99-109. MR,1711582 (2000i:16022)

[26] F. Xu, Representations of categories and their applications, J. Algebra 317 (2007), 153-183. MR2360144 (2008m:18024)

Department of Mathematics, University of Aberdeen, Aberdeen, AB24 3UE ScotLAND, UNITED KINGDOM

E-mail address: m.linckelmann@abdn.ac.uk

Department of Mathematical Sciences, University of Aberdeen, Aberdeen, AB24 3FX Scotland, United Kingdom

E-mail address: ro2mjs8@abdn.ac.uk 